\title{
CARACTERÍSTICAS DE LA LEUCEMIA LINFOBLÁSTICA AGUDA Y NEUTROPENIA FEBRIL EN NIÑOS Y ADOLESCENTES ATENDIDOS EN UN HOSPITAL DE GUAYAQUIL, ECUADOR
}

\author{
Lissette Fuentes $^{1, a}$, María Flores ${ }^{1, a}$, Adriana Iglesias ${ }^{1, a}$, Adriana Luzuriaga ${ }^{1, a}$, Nataly Rendón ${ }^{1, a}$, \\ Robert Ordoñez ${ }^{1, a}$, Fanny Solórzano ${ }^{1, b, c}$, Roberto Añez ${ }^{2, a}$
}

\begin{abstract}
RESUMEN
Con el objetivo de caracterizar clínicamente a los niños y adolescentes con leucemia linfoblástica aguda y neutropenia febril en un hospital de Ecuador se realizó un estudio de serie de casos revisando historias clínicas de pacientes atendidos en el Hospital de la Sociedad de Lucha Contra el Cáncer de la ciudad de Guayaquil durante enero 2014 y abril 2016. De los 101 pacientes $51,5 \%$ eran mujeres, la edad promedio fue $5,5 \pm 2,1$ años, el $72,3 \%$ tenían riesgo alto de infección bacteriana invasora, el foco de origen infeccioso más frecuente fue el respiratorio con $47,5 \%$, el $18,8 \%$ de los microorganismos aislados en hemocultivos fueron Gram-positivos. El $25,7 \%$ de los pacientes fallecieron, siendo la refractariedad a la quimioterapia uno de los factores asociados $(p<0,001)$. En conclusión, la mortalidad en niños y adolescentes, con leucemia linfoblástica aguda y neutropenia febril fue elevada; siendo la refractariedad a la quimioterapia uno de los principales factores asociados.
\end{abstract}

Palabras clave: Neutropenia febril; Leucemia linfoblástica; Mortalidad; Sepsis; Cáncer; Niños. (fuente: DeCS BIREME).

\section{CHARACTERISTICS OF ACUTE LYMPHOBLASTIC LEUKEMIA AND FEBRILE NEUTROPENIA IN CHILDREN AND ADOLESCENTS CARED FOR IN A HOSPITAL IN GUAYAQUIL, ECUADOR}

\begin{abstract}
With the objective of performing a clinical characterization of children and adolescents with acute lymphoblastic leukemia and febrile neutropenia in a hospital in Ecuador, a case series study was carried out, which involved reviewing the clinical records of patients receiving care at "Hospital de la Sociedad de Lucha Contra el Cancer" in Guayaquil between January 2014 and April 2016. Out of the 101 patients, 51.5\% were female; mean age was $5.5 \pm 2.1$ years old; $72.3 \%$ were at high risk for invasive bacterial infection; the most common infections were respiratory with $47.5 \% ; 18.8 \%$ of bacteria isolated in blood cultures were Gram-positive. Finally, $25.7 \%$ of patients died, with chemotherapy resistance as one of the associated factors.
\end{abstract}

Keywords: Febrile neutropenia; Lymphoblastic leukemia; Mortality; Sepsis; Cancer; Child. (source: MeSH NLM).

\section{INTRODUCCIÓN}

La leucemia linfoblástica aguda (LLA), es un tipo de cáncer que se inicia en los glóbulos blancos llamados linfocitos en la médula ósea, el término agudo hace referencia a que la enfermedad puede avanzar rápidamente y de no ser tratada podría ser letal al cabo de unos meses, mientras que linfocítico significa que se origina en las primeras etapas (inmaduras) de los linfocitos ${ }^{(1)}$. Esta constituye la neoplasia más común en menores de 15 años, con mayor incidencia entre los dos y cinco años de edad (2).

La característica principal de los pacientes con LLA, es la mayor predisposición a desarrollar infecciones por su estado de inmunodepresión, además de ser vulnerables al conocido efecto de la quimioterapia sobre el recuento

Universidad de Especialidades Espíritu Santo. Guayaquil. República del Ecuador.

Centro de Investigaciones Endocrino - Metabólicas - «Dr. Félix Gómez». Facultad de Medicina. La Universidad del Zulia. Maracaibo, Venezuela

Médico General; ${ }^{\mathrm{b}}$ Médico especialista en Pediatría; ${ }^{\mathrm{c}}$ Magister en Nutrición Clínica

Recibido: 05/05/2017 Aprobado: 21/02/2018 En línea: 15/05/2018

Citar como: Fuentes L, Flores M, Iglesias A, Luzuriaga A, Rendon N, Ordoñez R, et al. Características de la leucemia linfoblástica aguda y neutropenia febril en niños y adolescentes atendidos en un hospital de Guayaquil, Ecuador. Rev Peru Med Exp Salud Publica. 2018;35(2):272-8. doi: 10.17843/rpmesp.2018.352.2862. 
leucocitario y la consecuente aparición de fiebre, fenómeno conocido comúnmente como neutropenia febril, el cual es un factor frecuentemente asociado a mal pronóstico y al desarrollo de infección bacteriana invasora ${ }^{(3)}$. La neutropenia febril, se define como el recuentro absoluto de neutrófilos $(\mathrm{RAN})<1500$ células/ $\mu$ l pero, por razones de manejo en pacientes oncológicos se la define como el RAN $<500$ células/ $\mu$ l, además de un pico febril mayor de 38,5 ${ }^{\circ} \mathrm{C}$ o dos mayores a $38{ }^{\circ} \mathrm{C}$ en un intervalo no menor a una hora, tomados en axilas ${ }^{(4)}$.

Es importante resaltar que las infecciones en pacientes con neutropenia febril generan un impacto epidemiológico con una mayor morbimortalidad, aunado al aumento en costos debido a factores como internación, toma de exámenes diagnósticos, requerimiento de unidad de cuidados intensivos pediátricos (UCIP), uso de quirófanos para procedimientos invasivos y tratamiento antimicrobiano ${ }^{(5)}$.

El objetivo de este estudio fue caracterizar clínicamente a los niños y adolescentes ingresados con LLA y neutropenia febril en el Instituto Oncológico Nacional de Guayaquil, Ecuador.

\section{EL ESTUDIO}

Estudio de tipo descriptivo, retrospectivo en todos los pacientes con diagnóstico de LLA que ingresaron en el servicio de pediatría del Hospital de la Sociedad de Lucha Contra el Cáncer (SOLCA) de la ciudad de Guayaquil y que acudieron por cuadro clínico compatible con neutropenia febril y con riesgo de infección bacteriana invasora, en el periodo comprendido entre enero 2014 y abril 2016. Se excluyeron pacientes con más de un diagnostico oncológico, pacientes con infección viral o micótica como motivo de ingreso, paciente con registros incompletos.

Los grupos de edad se definieron según los intervalos propuesto por el Sistema de Indicadores Sociales del Ecuador. El uso de quimioterapia se definió, como el cumplimiento de una pauta de inducción o consolidación durante su estancia intrahospitalaria. El nivel de neutropenia se definió como neutropenia leve (Riesgo de infección): RAN $<1000$ células/ $\mu$ l, neutropenia moderada (Riesgo de infección mayor): RAN $<500$ células/ $\mu$ l y neutropenia profunda: RAN $<100$ células/ $\mu$ l. La sepsis se definió como sospecha clínica de infección y alguna evidencia de respuesta sistémica (taquicardia, taquipnea, hipertermia o hipotermia, leucocitosis o leucopenia, aumento del número de neutrófilos en cayado y de los reactantes de fase aguda en sangre periférica) ${ }^{(6)}$.

Por su parte, para efectos de manejo del paciente neutropénico febril y con el objetivo de dar un tratamiento ambulatorio o de hospitalización eficaz, se clasifica al momento del ingreso en bajo o alto riesgo y se reevalúa a las 48 a 72 horas ${ }^{(7)}$.

\section{MENSAJES CLAVE}

Motivación para realizar el estudio. En Ecuador y Latinoamérica existen escasos reportes que caractericen a los pacientes que padecen leucemia linfoblástica aguda y neutropenia febril durante el curso clínico de su enfermedad,

Principales hallazgos. La mortalidad en niños y adolescentes, con leucemia linfoblástica aguda y neutropenia febril fue elevada; los resultados muestran la importancia de la duración de la neutropenia, la presencia de sepsis y refractariedad a la quimioterapia; como los principales factores asociados

Implicancias. El elevado número de complicaciones, y la alta mortalidad en niños y adolescentes con leucemia linfoblástica aguda son factores que obligan al análisis periódico de las estrategias de abordaje empleadas para su reevaluación constante.

Riesgo bajo de infección: Al ingreso, buen estado general y nutricional, cáncer en remisión parcial o completa, predicción de neutropenia menor a diez días, sin signos de comorbilidad (sangrados, trastornos metabólicos refractarios, hipertensión arterial o endocraneana, distrés respiratorio), ausencia de focos infecciosos. A las 48-72 horas: Hemocultivos negativos, proteína $C$ reactiva $(P C R)$ que ha bajado por lo menos un $30 \%$, foco clínico controlado, afebril, sin signos de comorbilidad grave agregada.

Riesgo alto de infección: Al ingreso del paciente, estado general regular a malo y mal estado nutricional, enfermedad de base no controlada, leucemia en inducción o recaída, expectativa de neutropenia mayor de siete días y/o profunda, signos de comorbilidad no controlados, foco infeccioso presente, episodio de origen hospitalario o recaída de la fiebre, sepsis. Alas 48-72 horas: Hemocultivos positivos, aparición de signos de comorbilidad, foco clínico no controlado, aparición de signos clínicos, persistencia de la fiebre.

Todas las muestras de laboratorio fueron tomadas en las primeras 24 horas de evolución intrahospitalaria, los niveles de procalcitonina e interleucina 6 (IL-6) se detectaron por inmunoensayos de quimioluminiscencia y fueron medidos por el equipo Roche Elecsys E170 (Roche Diagnostics Gmbtt Mannheim, Germany). La PCR sérica se cuantificó por el sistema analítico proteico IMMAGE 800 (Beckman Coulter company, USA). Los cultivos bacterianos se realizaron en el laboratorio de microbiología de nuestro hospital, todas las muestras se tomaron con las medidas de asepsia respectivas.

Las variables cualitativas fueron presentadas como frecuencias absolutas y relativas (porcentaje), se utilizó la prueba de chi cuadrado de Pearson para determinar la asociación entre variables cualitativas, se consideraron los resultados estadísticamente significativos cuando $p<0,05$. Se utilizó el programa Statistical Package for the Social Sciences (SPSS) versión 22. 
El estudio tuvo la autorización del comité de bioética y docencia del Hospital SOLCA.

\section{RESULTADOS}

Se incluyeron 101 pacientes el 51,5\% ( $n=51)$ fueron del sexo femenino. El grupo etario más frecuente fue de dos a cinco años con 49,5\% ( $n=50)$, mientras que un $91,1 \%$ $(n=92)$ recibieron quimioterapia durante su estancia intrahospitalaria. Por su parte, un $64,4 \%(n=65)$ presentaron niveles de PCR $<20 \mathrm{mg} / \mathrm{dL}, 81,2 \%$ ( $n=82)$ mostraron cifras de plaquetas entre cinco y $150 \times 10 \% / L ; 56,9 \%(n=33)$ tuvieron procalcitonina $>2 \mathrm{ng} / \mathrm{ml}$ y hasta un $77,5 \%(\mathrm{n}=55)$ presentaron valores de IL-6 $\geq 7 \mathrm{pg} / \mathrm{ml}$. El 13,9\% $(\mathrm{n}=14)$ presentaron sepsis y la mortalidad fue de $25,7 \%(n=26)$ (Tabla 1$)$.

En los cultivos de secreciones los microorganismos Gramnegativos fueron los más aislados con un 26,7\% ( $n=27)$, mientras que en los hemocultivos los microorganismo Grampositivos fueron los más encontrados con 18,8\% $(n=19)$ y en los urocultivos se hallaron hasta un $34,7 \%(n=35)$ de bacterias Gram-negativas (Tabla 2).

Tabla 1. Características de pacientes con leucemia linfoblástica aguda en el Instituto Oncológico Nacional, Guayaquil, Ecuador

\begin{tabular}{|c|c|c|c|c|c|c|}
\hline & \multicolumn{2}{|c|}{ Femenino } & \multicolumn{2}{|c|}{ Masculino } & \multicolumn{2}{|c|}{ Total } \\
\hline & $\mathrm{n}=51$ & $\%$ & $\mathrm{n}=50$ & $\%$ & $\mathrm{n}=101$ & $\%$ \\
\hline \multicolumn{7}{|c|}{ Grupos etarios (años) } \\
\hline $2-5$ & 24 & 47,1 & 26 & 52,0 & 50 & 49,5 \\
\hline $6-11$ & 18 & 35,3 & 13 & 26,0 & 31 & 30,7 \\
\hline $12-18$ & 9 & 17,6 & 11 & 22,0 & 20 & 19,8 \\
\hline \multicolumn{7}{|c|}{ Recibió quimioterapia } \\
\hline No & 4 & 7,8 & 5 & 10,0 & 9 & 8,9 \\
\hline Sí & 47 & 92,2 & 45 & 90,0 & 92 & 91,1 \\
\hline \multicolumn{7}{|c|}{ Refractario a quimioterapia } \\
\hline No & 38 & 80,9 & 39 & 86,7 & 77 & 83,7 \\
\hline Sí & 9 & 19,1 & 6 & 13,3 & 15 & 16,3 \\
\hline \multicolumn{7}{|c|}{ 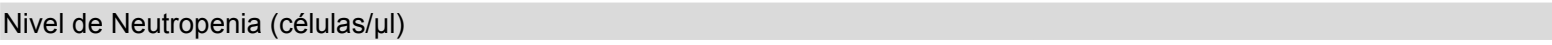 } \\
\hline$<1000$ & 8 & 15,7 & 2 & 4,0 & 10 & 9,9 \\
\hline$<500$ & 15 & 29,4 & 14 & 28,0 & 29 & 28,7 \\
\hline$<100$ & 28 & 54,9 & 34 & 68,0 & 62 & 61,4 \\
\hline \multicolumn{7}{|c|}{ Duración de neutropenia (días) } \\
\hline$<7$ & 15 & 29,4 & 10 & 20,0 & 25 & 24,8 \\
\hline 7 o más & 36 & 70,6 & 40 & 80,0 & 76 & 75,2 \\
\hline \multicolumn{7}{|c|}{ Riesgo de infección } \\
\hline Bajo & 15 & 29,4 & 13 & 26,0 & 28 & 27,7 \\
\hline Alto & 36 & 70,6 & 37 & 74,0 & 73 & 72,3 \\
\hline \multicolumn{7}{|c|}{ Proteína C Reactiva (mg/dL) } \\
\hline$<20$ & 31 & 60,8 & 34 & 68,0 & 65 & 64,4 \\
\hline $20-40$ & 17 & 33,3 & 14 & 28,0 & 31 & 30,7 \\
\hline$>40$ & 3 & 5,9 & 2 & 4,0 & 5 & 5,0 \\
\hline \multicolumn{7}{|c|}{ Plaquetas (x109/L) } \\
\hline$>150$ & 7 & 13,7 & 7 & 14,0 & 14 & 13,9 \\
\hline $5-150$ & 43 & 84,3 & 39 & 78,0 & 82 & 81,2 \\
\hline$<5$ & 1 & 2,0 & 4 & 8,0 & 5 & 5,0 \\
\hline \multicolumn{7}{|c|}{ Procalcitonina (ng/ml) } \\
\hline$<1$ & 12 & 37,5 & 11 & 42,3 & 23 & 39,7 \\
\hline $1-2$ & 2 & 6,3 & 0 & 0,0 & 2 & 3,4 \\
\hline$>2$ & 18 & 56,3 & 15 & 57,7 & 33 & 56,9 \\
\hline \multicolumn{7}{|c|}{ Interleucina -6 (pg/ml) } \\
\hline$<7$ & 9 & 23,1 & 7 & 21,9 & 16 & 22,5 \\
\hline$\geq 7$ & 30 & 76,9 & 25 & 78,1 & 55 & 77,5 \\
\hline \multicolumn{7}{|l|}{ Sepsis } \\
\hline No & 43 & 84,3 & 44 & 88,0 & 87 & 86,1 \\
\hline Sí & 8 & 15,7 & 6 & 12,0 & 14 & 13,9 \\
\hline \multicolumn{7}{|l|}{ Fallecido } \\
\hline No & 34 & 66,7 & 41 & 82,0 & 75 & 74,3 \\
\hline Sí & 17 & 33,3 & 9 & 18,0 & 26 & 25,7 \\
\hline
\end{tabular}


Tabla 2. Características bacteriológicas según tipo de cultivo en niños y adolescentes con leucemia linfoblástica aguda y neutropenia febril. Instituto Oncológico Nacional, Guayaquil, Ecuador

\begin{tabular}{lcc}
\hline Tipo de microorganismo & $\mathbf{n = 1 0 1}$ & $\%$ \\
\hline Cultivos de secreciones & & \\
$\quad$ No aislado & 71 & 70,3 \\
Gram-negativo & 27 & 26,7 \\
Gram-positivo & 2 & 2,0 \\
$\quad$ Gram-positivo + Gram-negativo & 1 & 1,0 \\
\hline Hemocultivos & & \\
$\quad$ No aislado & 61 & 60,4 \\
\hline Gram-positivo & 19 & 18,8 \\
Gram negativo & 17 & 16,8 \\
\hline Gram-positivo + Gram-negativo & 4 & 4,0 \\
Urocultivos & & \\
$\quad$ No aislado & 66 & 65,3 \\
$\quad$ Gram-negativo & 35 & 34,7 \\
\hline
\end{tabular}

El origen infeccioso más frecuente fue el respiratorio con $47,5 \%(n=48)$, seguido de la combinación respiratorio y gastrointestinal como origen $8,9 \%(n=9)$. Según la frecuencia en el uso de antibióticos los aminoglucósidos fueron los más empleados con $83,2 \%$ ( $n=84)$; seguido de los carbapenémicos
( $n=63)$ y glucopéptidos $(n=63)$ ambos con $62,4 \%$, cefalosporinas $51,5 \%(n=52)$, penicilinas $32,7 \% \quad(n=33)$, fluoroquinolonas 11,9\% ( $n=12)$, Trimetropim-Sulfametoxazol $8,9 \%(n=9)$, metronidazol $(n=5) 5,0 \%$, macrólidos 4,0\% $(n=4)$, glicilciclinas $2,0 \%(n=2)$ y colistina $1,0 \%(n=1)$.

En la Tabla 3 se muestra la distribución según infección bacteriana invasora con porcentajes más elevados en los pacientes con mayor nivel de neutropenia $(p<0,001)$, niveles más altos de PCR $(p=0,04)$ y mayor duración de la neutropenia $(p<0,001)$.

Los factores asociados a mortalidad fueron la refractariedad a la quimioterapia $(p<0,001)$ y el riesgo de infección $(p=0,002)$, asimismo el mayor grupo etario $(p=0,002)$, y la presencia de sepsis $(p<0,001)$ (Tabla 4).

\section{DISCUSIÓN}

En este estudio se muestra como ambas entidades clínicas generan una alta mortalidad, asociado a la presencia de refractariedad a la quimioterapia, sepsis y riesgo alto de infección.

La alta frecuencia de neutropenia febril en los pacientes de edad preescolar, podría estar relacionada a la mayor

Tabla 3. Distribución según infección bacteriana invasora en niños y adolescentes con leucemia linfoblástica aguda y neutropenia febril. Instituto Oncológico Nacional, Guayaquil, Ecuador

\begin{tabular}{|c|c|c|c|c|c|}
\hline & \multicolumn{4}{|c|}{ Infección bacteriana invasora } & \multirow{3}{*}{ Valor de $p$} \\
\hline & \multicolumn{2}{|c|}{ Bajo } & \multicolumn{2}{|c|}{ Alto } & \\
\hline & $\mathrm{n}=\mathbf{2 8}$ & $\%$ & $n=73$ & $\%$ & \\
\hline \multicolumn{6}{|l|}{ Sexo } \\
\hline Masculino & 13 & 46,4 & 37 & 50,7 & 0,702 \\
\hline Femenino & 15 & 53,6 & 36 & 49,3 & \\
\hline \multicolumn{6}{|c|}{ Grupos etarios (años) } \\
\hline $2-5$ & 15 & 53,6 & 35 & 47,9 & 0,693 \\
\hline $6-11$ & 9 & 32,1 & 22 & 30,1 & \\
\hline $12-18$ & 4 & 14,3 & 16 & 21,9 & \\
\hline \multicolumn{6}{|c|}{ Refractario a quimioterapia $(\mathrm{n}=92)$} \\
\hline No & 21 & 91,3 & 56 & 81,2 & 0,255 \\
\hline Sí & 2 & 8,7 & 13 & 18,8 & \\
\hline \multicolumn{6}{|c|}{ Nivel de Neutropenia (células/ $\mu \mathrm{l}$ ) } \\
\hline$<1000$ & 7 & 25,0 & 3 & 4,1 & $<0,001$ \\
\hline$<500$ & 13 & 46,4 & 16 & 21,9 & \\
\hline$<100$ & 8 & 28,6 & 54 & 74,0 & \\
\hline \multicolumn{6}{|c|}{ Nivel de Proteína C Reactiva (mg/dL) } \\
\hline$<20$ & 25 & 89,3 & 40 & 54,8 & 0,004 \\
\hline $20-40$ & 2 & 7,1 & 29 & 39,7 & \\
\hline$>40$ & 1 & 3,6 & 4 & 5,5 & \\
\hline \multicolumn{6}{|c|}{ Duración de Neutropenia (días) } \\
\hline$<7$ & 16 & 57,1 & 9 & 12,3 & $<0,001$ \\
\hline$\geq 7$ & 12 & 42,9 & 64 & 87,7 & \\
\hline
\end{tabular}


Tabla 4. Distribución según mortalidad en niños y adolescentes con leucemia linfoblástica aguda y neutropenia febril. Instituto Oncológico Nacional, Guayaquil, Ecuador

\begin{tabular}{|c|c|c|c|c|c|}
\hline & \multicolumn{4}{|c|}{ Mortalidad } & \multirow{3}{*}{ Valor de $p$} \\
\hline & \multicolumn{2}{|c|}{ No } & \multicolumn{2}{|c|}{ Sí } & \\
\hline & $n=75$ & $\%$ & $\mathrm{n}=\mathbf{2 6}$ & $\%$ & \\
\hline \multicolumn{6}{|l|}{ Sexo } \\
\hline Niños & 41 & 54,7 & 9 & 34,6 & 0,078 \\
\hline Niñas & 34 & 45,3 & 17 & 65,4 & \\
\hline \multicolumn{6}{|c|}{ Grupos etarios (años) } \\
\hline $2-5$ & 43 & 57,3 & 7 & 26,9 & 0,002 \\
\hline $6-11$ & 23 & 30,7 & 8 & 30,8 & \\
\hline $12-18$ & 9 & 12,0 & 11 & 42,3 & \\
\hline \multicolumn{6}{|c|}{ Refractario a quimioterapia } \\
\hline No & 64 & 97,0 & 13 & 50,0 & $<0,001$ \\
\hline Sí & 2 & 3,0 & 13 & 50,0 & \\
\hline \multicolumn{6}{|c|}{ Riesgo de infección } \\
\hline Bajo & 27 & 36,0 & 1 & 3,8 & 0,002 \\
\hline Alto & 48 & 64,0 & 25 & 96,2 & \\
\hline \multicolumn{6}{|l|}{ Sepsis } \\
\hline No & 70 & 93,3 & 17 & 65,4 & $<0,001$ \\
\hline Sí & 5 & 6,7 & 9 & 34,6 & \\
\hline
\end{tabular}

vulnerabilidad inmune, que ante el insuficiente desarrollo y el efecto de la quimioterapia condicionan una pobre respuesta ante infecciones. Los resultados varían de otro estudio realizado previamente en Ecuador donde se encontró que la frecuencia era mayor en adolescentes (35\%), seguidos por preescolares $(34 \%)^{(8)}$. Mientras que un reporte sobre la caracterización de los episodios de neutropenia febril en niños con cáncer en Colombia muestra que los más afectados son los niños de tres años $(17 \%)^{(9)}$.

En cuanto a los parámetros de laboratorio, la mayor proporción de pacientes presentaron neutropenia grave, similar a lo planteado por Suarez y cols. ${ }^{(10)}$, en un hospital en Colombia. No obstante, no solo la severidad de la neutropenia inicialmente es de importancia, la duración de la misma también parece constituir un factor pronóstico no sólo para neoplasias de origen hematológico sino también para tumores sólidos ${ }^{(11)}$. Por su parte, similar a lo planteado en la población colombiana donde todos fueron considerados de alto riesgo de infección bacteriana invasora, el porcentaje de pacientes con esta característica fue mayor del $70 \%$, lo cual demuestra la necesidad de ser manejados de manera intensiva a nivel intrahospitalario. Entre los mediadores inflamatorios de fase aguda, la procalcitonina y la IL-6 constituyen las moléculas que mostraron niveles más elevados en este grupo de pacientes, las cuales varían en las diversas series representando un importante tema de análisis en la infectología pediátrica actualmente ${ }^{(12,13)}$.

Otro aspecto que muestra una amplia variabilidad es el origen de la infección, similar a los hallazgos Arnello y cols. ${ }^{(14)}$, donde el origen de las infecciones bacterianas fue respiratorio (19,3\%), cutánea (10\%) y digestiva (7\%) en 57 episodios de neutropenia febril en 25 pacientes chilenos. Asimismo, en los pacientes pediátricos colombianos los síntomas principales son los respiratorios y hasta un $31 \%$ no presentaron síntomas ${ }^{(10)}$. En un estudio previo realizado en Ecuador, donde se evaluó el comportamiento de neutropenia febril en leucemia aguda, se encontró que las tres patologías determinantes de mal pronóstico y evolución fueron: celulitis, mucositis e infecciones respiratorias ${ }^{(9)}$.

Los microorganismos aislados varían de acuerdo al tipo de cultivo, en el caso de los hemocultivos los resultados coinciden con los reportados en las diversas series en las últimas décadas, donde ha ocurrido una inversión en la tendencia epidemiológica de bacterias Gram-negativas a Gram-positivas ${ }^{(15)}$. No obstante, la diferencia entre ambos grupos en nuestro análisis no fue tan marcada, diferencias que han sido reportadas previamente en los pacientes estudiados (16); en relación a los cultivos de secreción y urocultivos el predominio de bacterias Gram-negativas es un importante hallazgo a considerar para el inicio de la antibioticoterapia empírica en este grupo de sujetos.

Pese al predominio de las infecciones del tracto respiratorio, de la presencia de microorganismos Gram-positivos en hemocultivos y de la baja prevalencia de sepsis en la muestra estudiada, resulta interesante observar que el principal grupo de antibióticos empleados fueron los aminoglucósidos, los cuales figuran entre las opciones de tratamiento cuando sea necesaria la combinación de fármacos, cuando exista inestabilidad clínica o ante una alta tasa de resistencia ${ }^{(17)}$. Esto demuestra la necesidad 
de reevaluar los protocolos de manejo en nuestra región y ajustarlos a las características propias exhibidas en nuestra población.

La mortalidad relacionada a sepsis fue superior a la mostrada en diversos reportes donde oscila entre el 9,3\% en niños con LLAen tres países de Centroamérica ${ }^{(18)}$ y menos de $2 \%$ según revisiones sistemáticas ${ }^{(19)}$. En este sentido, identificar los principales factores asociados puede contribuir a tomar un abordaje y manejo más eficaz según cada paciente. El mayor riesgo en el sexo femenino es un hallazgo particular en nuestra población, pudiendo relacionarse a la mayor refractariedad a la quimioterapia e infección por microorganismos más resistentes a la antibioticoterapia, mientras que el mayor riesgo en los adolescentes en comparación con edades más tempranas también ha sido planteado por Basu y cols. ${ }^{(20)}$. De igual manera, la presencia de sepsis y la refractariedad a la quimioterapia, especialmente en niños con LLA, son factores descritos en otras poblaciones de Latinoamérica ${ }^{(21)}$ y África ${ }^{(22)}$.

El riesgo de infección bacteriana invasora es otro parámetro que debe ser evaluado de forma exhaustiva al ingreso hospitalario, dada su estrecha relación con la mortalidad a corto plazo, especialmente en pacientes cuya duración de la neutropenia se extienda por más de siete días, los cuales deben ser considerados pacientes de alto riesgo y según las recomendaciones actuales se plantea el inicio de antibioticoterapia profiláctica con quinolonas en adultos ${ }^{(23)}$, mientras que en niños el tema es más controvertido y en la actualidad se discuten las ventajas y desventajas de este uso preventivo ${ }^{(24)}$. En vista de los hallazgos y la estrecha relación con mortalidad sugerimos el uso de antibioticoterapia en este grupo de pacientes, la cual estará destinada al microorganismo aislado en el cultivo inicial, siendo los Gram-positivos los más frecuentes en nuestra población o tomando en cuenta los patrones de resistencia local.

Entre las limitaciones del estudio se encuentra el carácter retrospectivo de la investigación y el número limitado de pacientes, dependiente de una fuente secundaria y de los ingresos durante el periodo analizado, lo cual genera posibles sesgos en la interpretación; así como la evaluación de otro tipo de neoplasias que pudieran constituir factores de confusión.

En conclusión, los hallazgos muestran que la mortalidad es elevada en niños y adolescentes con LLA y neutropenia febril atendidos en el Instituto Oncológico Nacional de Guayaquil; siendo la refractariedad a la quimioterapia, el riesgo alto de infección y la presencia de sepsis los principales factores asociados, resaltando la necesidad de identificar de manera temprana y oportuna los cuadros infecciosos.

Contribuciones de autoría: LF participó en la concepción, diseño del artículo y recolección de resultados, MF participó en la concepción, diseño del artículo y redacción del artículo, Al participó en la recolección de resultados y redacción del artículo, AL participó en la recolección de resultados y asesoría estadística, NR, RO participaron en el análisis e interpretación de datos y revisión crítica del artículo. Todos los autores aprobaron la versión final.

Fuentes de financiamiento: Autofinanciado

Conflictos de interés: Los autores declaran no tener conflictos de interés.

\section{REFERENCIAS BIBLIOGRÁFICAS}

1. Ribera JM, Tormo M, Ortega JJ.Leucemia linfoblástica aguda en adolescentes y adultos jóvenes. Med Clin Monogr (Barc) 2007;129(Supl 1):36-9.

2. Ortega M, Osnaya M, Rosas J. Leucemia linfoblástica aguda. Med Int Mex 2007;23:26-33.

3. Lehrnbecher T, Foster C, Vázquez N, Mackall CL, Chanock SJ. Therapyinduced alterations in host defense in children receiving therapy for cancer. J Pediatr Hematol Oncol. 1997; 19(5):399-417.

4. Pérez P, Pérez E. Neutropenia febril en pediatría. Infectio. 2008;12(1):64-71.

5. Ahmed N, El-Mahallawy HA, Ahmed IA, Nassif S, El-Beshlawy A, El-Haddad A. Early hospital discharge versus continued hospitalization in febrile pediatric cancer patients with prolonged neutropenia: A randomized, prospective study. Pediatr Blood Cancer. 2007;49(6):786-92.

6. Dellinger RP, Levy MM, Rhodes A, Annane D, Gerlach H, Opal SM, et al. Surviving Sepsis Campaign Guidelines Committee including the Pediatric Subgroup. Surviving sepsis campaign: international guidelines for management of severe sepsis and septic shock: 2012. Crit Care Med. 2013;41(2):580-637.

7. Paganini $\mathrm{H}$, Santolaya ME, Álvarez M, Araña M, Arteaga R, Bonilla A, et al. Diagnóstico y tratamiento de la neutropenia febril en niños con cáncer. Consenso de la Sociedad Latinoamericana de Infectología Pediátrica. Rev Chil Infect 2011; 28 (Supl 1): 10-38.

8. Curado MP, Pontes T, Guerra-Yi ME, de Camargo Cancela M. Leukemia mortality trends in children, adolescents, and young adults in Latin America. Rev Panam Salud Pública. 2011;29(2):96-102.

9. Rincón C. Caracterización de episodios de neutropenia febril en niños con cáncer en la Fundación Hospital de la Misericordia. [Tesis conducente al título de Pediatra]. Consultado: 21/04/17. Disponible: http://www.bdigital.unal. edu.co/11407/1/598779.2013.pdf

10. Suarez DV, Álvarez M, Gómez JF, Carrasco MM, Burbano DC. Caracterización clínica y de laboratorio de pacientes con neutropenia febril en un hospital pediátrico en Pasto-Colombia. Rev Col Ped 2016;49:48-53.

11. Caggiano V, Weiss RV, Rickert TS, Linde-Zwirble WT. Incidence, cost, and mortality of neutropenia hospitalization associated with chemotherapy. Cancer. 2005;103(9):1916-24. 
12. Phillips RS, Wade R, Lehrnbecher T, Stewart LA, Sutton AJ. Systematic review and meta-analysis of the value of initial biomarkers in predicting adverse outcome in febrile neutropenic episodes in children and young people with cancer. BMC Med. 2012;10:6. doi: 10.1186/17417015-10-6.

13. Urbonas V, Eidukaite A. Diagnostic accuracy of procalcitonin, interleukin- 6 and interleukin- 8 for predicting Gramnegative bacteremia in febrile neutropenia patients with acute lymphoblastic leukemia. Acta Med Litu. 2014;21.(4): 178-181.

14. Arnello M, Quintana JA, Barraza P. Neutropenia febril en niños con cáncer en un centro asistencial en Santiago, Chile. Rev Chil Infect 2007; 24 (1): 27-32.

15. Dufort,G. Guía para el tratamiento del paciente con neutropenia febril. Arch Pediatr Urug, Arch Pediatr Urug 2009; 80(1):37-41.

16. BonillaA,BonillaH.PerfilMicrobiológico de las infecciones en pediatría del ION SOLCA Guayaquil. Año 2011. Consultado: 21/04/17. Disponible: http://hospitalalcivar.com/uploads/pdf/ Perfil\%20microbiol\%C3\%B3gico\%20 de\%20las\%20infecciones\%20en $\% 20$ pediatr\%C3\%ADa\%202015.pdf

17. Lehrnbecher T, Phillips R, Alexander S, Alvaro F, Carlesse F, et al. Guideline for the management of fever and neutropenia in children with cancer and/or undergoing hematopoietic stem-cell transplantation. J Clin Oncol. 2012;30(35):4427-38.

18. Gupta S, Antillon FA, Bonilla M, Fu L, Howard SC, Riberiro RC, et al. Treatment-Related Mortality in Children With Acute Lymphoblastic Leukemia in Central America. Cancer. 2011;117(20):4788-95.

19. Phillips RS, Sutton AJ, Riley RD, Chisholm JC, Picton SV, Stewart LA. Predicting infectious complications in neutropenic children and young people with cancer (IPD protocol). Syst Rev. 2012; 1: 8 .

20. Basu SK, Fernandez ID, Fisher SG, Asselin BL, Lyman GH. Length of stay and mortality associated with febrile neutropenia among children with cancer. J Clin Oncol. 2005;23(31):7958-66.

21. Santolaya ME. Alvarez AM, Avilés C, Becker A, Mosso C, O'Ryan M, et al. Admission Clinical and Laboratory Factors Associated With Death in
Children With Cancer During a Febrile Neutropenic Episode. Pediatr Infect Dis J 2007;26: 794-798.

22. Badr M, Hassan T, Sakr H, Karam N, Rahman DA, Shahbah D, et al. Chemotherapy-induced neutropenia among pediatric cancer patients in Egypt: Risks and consequences. Mol Clin Oncol. 2016; 5(3): 300-306.

23. Villafuerte-Gutiérrez P, Villalon L, Losa JE, Henriquez-Camacho C. Treatment of Febrile Neutropenia and Prophylaxis in Hematologic Malignancies: A Critical Review and Update. Adv Hematol. 2014; 2014: 986938.

24. Alexander S, Nieder M, Zerr DM, Fisher BT, Dvorak CC, Sung L. Prevention of bacterial infection in pediatric oncology: what do we know, what can we learn? Pediatr Blood Cancer. 2012;59(1):16-20.

Correspondencia: Lissette Nathalie Fuentes, MD. Universidad de Especialidades Espiritu Santo. Guayaquil - Ecuador. Av. Samborondón, Samborondón 092301, Guayaquil. República del Ecuador. Teléfono: +593996543223 Correo electrónico:lissnathali@gmail.com 